\title{
Weakly useful sequences
}

\author{
Stephen A. Fenner ${ }^{1}$ \\ University of South Carolina, Columbia, South Carolina, USA \\ E-mail: fenner@cse.sc.edu \\ Jack H. Lutz ${ }^{2}$ \\ Iowa State University, Ames, Iowa, USA \\ E-mail: lutz@iastate.edu \\ Elvira Mayordomo ${ }^{3}$ \\ University of Zaragoza, Zaragoza, SPAIN \\ E-mail: elvira@unizar.es \\ Patrick Reardon ${ }^{4}$ \\ SE Oklahoma State University, Durant, Oklahoma, USA \\ E-mail: preardon@sosu.edu
}

\begin{abstract}
An infinite binary sequence $x$ is defined to be

(i) strongly useful if there is a computable time bound within which every decidable sequence is Turing reducible to $x$; and

(ii) weakly useful if there is a computable time bound within which all the sequences in a non-measure 0 subset of the set of decidable sequences are Turing reducible to $x$.
\end{abstract}

Juedes, Lathrop, and Lutz (1994) proved that every weakly useful sequence is strongly deep in the sense of Bennett (1988) and asked whether there are sequences that are weakly useful but not strongly useful.

The present paper answers this question affirmatively. The proof is a direct construction that combines the martingale diagonalization technique of Lutz (1994) with a new technique, namely, the construction of a sequence that is "computably deep" with respect to an arbitrary, given uniform reducibility. The abundance of such computably deep sequences is also proven and used to show that every weakly useful sequence is computably deep with respect to every uniform reducibility. 
Key words: Computability, Randomness, Random sequence, Computational depth, Logical depth, Computable measure, Resource-bounded measure, Useful, Weakly useful

\section{Introduction}

It is a truism that the usefulness of a data object does not vary directly with its information content. For example, consider two infinite binary strings, $\chi_{K}$, the characteristic sequence of the halting problem (whose $n$th bit is 1 if and only if the $n$th Turing machine halts on input $n$ ), and $z$, a sequence that is algorithmically random in the sense of Martin-Löf [1]. The following facts are well-known.

(1) The first $n$ bits of $\chi_{K}$ can be specified using only $O(\log n)$ bits of information, namely, the number of 1's in the first $n$ bits of $\chi_{K}[2]$.

(2) The first $n$ bits of $z$ cannot be specified using significantly fewer than $n$ bits of information [1].

(3) Oracle access to $\chi_{K}$ would enable one to decide any decidable sequence in polynomial time (i.e., decide the $n$th bit of the sequence in time polynomial in the length of the binary representation of $n$ ) [3].

(4) Even with oracle access to $z$, most decidable sequences cannot be computed in polynomial time. (This appears to be folklore, known at least since [4].)

Facts (1) and (2) tell us that $\chi_{K}$ contains far less information than $z$. In contrast, facts (3) and (4) tell us that $\chi_{K}$ is computationally much more useful than $z$. That is, the information in $\chi_{K}$ is "more usefully organized" than that in $z$.

Bennett [4] introduced the notion of computational depth (also called "logical depth") in order to quantify the degree to which the information in an object has been organized. In particular, for infinite binary sequences, Bennett defined two "levels" of depth, strong depth and weak depth, and argued that the

1 Supported in part by National Science Foundation Grants CCR-9209833, CCR9501794, and CCR-9996310.

2 Supported in part by National Science Foundation Grants 9157382, 9610461, 9988483, and 0344187.

3 Supported in part by Spanish Government projects PB98-0937-C04-02 and TIC2002-04019-C03-03 and by National Science Foundation Grant 0344187.

4 Supported in part by a grant from the Applied and Organized Research Fund at SEOSU. 
above situation arises from the fact that $\chi_{K}$ is strongly deep, while $z$ is not even weakly deep. (The present paper is motivated by the study of computational depth, but does not directly use strong or weak depth, so definitions are omitted here. The interested reader is referred to [4], [5], or [6] for details, and for related aspects of algorithmic information theory.)

Investigating this matter further, Juedes, Lathrop, and Lutz [6] considered two "levels of usefulness" for infinite binary sequences. Specifically, let $\mathbf{C}$ be the Cantor space of all infinite binary sequences and let DEC be the set of all decidable elements of $\mathbf{C}$. For $x \in \mathbf{C}$ and $t: \mathbb{N} \rightarrow \mathbb{N}$, let $\operatorname{DTIME}^{x}(t)$ be the set of all $y \in \mathbf{C}$ for which there exists an oracle Turing machine $M$ that, on input $n \in \mathbb{N}$ with oracle $x$, computes $y[n]$, the $n$th bit of $y$, in at most $t(\ell)$ steps, where $\ell$ is the number of bits in the binary representation of $n$. Then a sequence $x \in \mathbf{C}$ is defined to be strongly useful if there is a computable time bound $t: \mathbb{N} \rightarrow \mathbb{N}$ such that $\operatorname{DTIME}^{x}(t)$ contains every decidable sequence. A sequence $x \in \mathbf{C}$ is defined to be weakly useful if there is a computable time bound $t: \mathbb{N} \rightarrow \mathbb{N}$ such that the set of decidable sequences contained in $\operatorname{DTIME}^{x}(t)$ is a non-measure 0 subset of DEC in the sense of resource-bounded measure [7]. That is, $x$ is weakly useful if access to $x$ enables one to decide a nonnegligible set of decidable sequences within some fixed computable time bound. No decidable or algorithmically random sequence can be weakly useful. It is evident that $\chi_{K}$ is strongly useful, and that every strongly useful sequence is weakly useful.

Juedes, Lathrop, and Lutz [6] generalized Bennett's result that $\chi_{K}$ is strongly deep by proving that every weakly useful sequence is strongly deep. This confirmed Bennett's intuitive arguments by establishing a definite relationship between computational depth and computational usefulness. It also substantially extended Bennett's result on $\chi_{K}$ by implying (in combination with known results of recursion theory [1,8-10]) that all high Turing degrees and some low Turing degrees contain strongly deep sequences.

Notwithstanding this progress, Juedes, Lathrop, and Lutz [6] left a critical question open: Do there exist weakly useful sequences that are not strongly useful? The main result of the present paper answers this question affirmatively. This establishes the existence of strongly deep sequences that are not strongly useful. More importantly, it indicates a need for further investigation of the class of weakly useful sequences.

The proof of our main result is a direct construction that combines the martingale diagonalization technique introduced by Lutz [11] with a new technique, namely, the construction of a sequence that is computably $F$-deep, where $F$ is an arbitrary uniform reducibility. This notion of computable uniform depth is closely related to Bennett's notion of weak depth. 
The paper is organized as follows. Section 2 contains basic definitions. In Section 3 we introduce and investigate the notions of computable $F$-depth and computable weak depth. In addition to using specific constructions of computably $F$-deep sequences, we prove that for each uniform reducibility $F$, almost every sequence in DEC is computably $F$-deep. This implies that a weakly useful sequence is computably $F$-deep for any uniform reducibility $F$. The main theorem is proved in Section 4, where in addition we introduce a canonical technique for constructing computably $F$-deep sequences that satisfy an additional property which, loosely translated, guarantees that the depths

of their initial segments increase at a rate exponential in the length of the segment.

\section{Preliminaries}

We use $\mathbb{N}$ to denote the set of natural numbers (including 0), and $\mathbb{Q}$ to denote the set of rational numbers. We write $\llbracket \varphi \rrbracket$ for the Boolean value of a condition $\varphi$, i.e.,

$$
\llbracket \varphi \rrbracket=\text { if } \varphi \text { then } 1 \text { else } 0
$$

For any $x, y \in\{0,1\}^{*} \cup\{0,1\}^{\infty}$, we write $x \sqsubseteq y$ to mean that $x$ is a prefix of $y$. For every $w \in\{0,1\}^{*}$, define $\mathbf{C}_{w}=\{x \in \mathbf{C}: w \sqsubseteq x\}$. We fix a computable, bijective pairing function $\langle\cdot, \cdot\rangle: \mathbb{N}^{2} \rightarrow \mathbb{N}$, monotone in both arguments, such that $i \leq\langle i, j\rangle$ and $j \leq\langle i, j\rangle$ for all $i, j \in \mathbb{N}$.

Weakly useful sequences are defined (in Section 1) in terms of computable measure, a special case of the resource-bounded measure developed by Lutz [7]. We very briefly sketch the elements of this theory, referring the reader to $[7,11]$ for motivation, details, and intuition.

Definition 1 A martingale is a function $d:\{0,1\}^{*} \rightarrow[0, \infty)$ such that $d(w)=$ $(d(w 0)+d(w 1)) / 2$ for all $w \in\{0,1\}^{*}$. A martingale $d$ is computable if there is a total computable function $\hat{d}: \mathbb{N} \times\{0,1\}^{*} \rightarrow \mathbb{Q}$ such that, for all $r \in \mathbb{N}$ and $w \in\{0,1\}^{*}$,

$$
|\hat{d}(r, w)-d(w)| \leq 2^{-r}
$$

We make use of two notions of "success" for a martingale.

Definition 2 Suppose $d$ is a martingale. 
(1) $d$ succeeds on a sequence $x \in \mathbf{C}$ if

$$
\limsup _{n \rightarrow \infty} d(x[0 \ldots n-1])=\infty,
$$

where $x[0 \ldots n-1]$ is the $n$-bit prefix of $x$.

(2) The success set of $d$ is

$$
S^{\infty}[d]=\{x \in \mathbf{C}: d \text { succeeds on } x\} .
$$

(3) The strong unitary success set of $d$ is

$$
S S^{1}[d]=\{x \in \mathbf{C}: \text { for all but finitely many } n, d(x[0 \ldots n-1]) \geq 1\} .
$$

Definition 3 Let $X \subseteq \mathbf{C}$.

(1) $X$ has computable measure 0 , and we write $\mu_{\text {comp }}(X)=0$, if there is a computable martingale $d$ such that $X \subseteq S^{\infty}[d]$.

(2) $X$ has computable measure 1 , and we write $\mu_{\text {comp }}(X)=1$, if $\mu_{\text {comp }}\left(X^{c}\right)=$ 0 , where $X^{c}=\mathbf{C}-X$ is the complement of $X$.

(3) $X$ has measure 0 in DEC, and we write $\mu(X \mid \mathrm{DEC})=0$, if $\mu_{\mathrm{comp}}(X \cap$ $\mathrm{DEC})=0$.

(4) $X$ has measure 1 in DEC, and we write $\mu(X \mid \mathrm{DEC})=1$, if $\mu\left(X^{c} \mid\right.$ $\mathrm{DEC})=0$. In this case, we say that $X$ contains almost every element of DEC.

\section{$3 \quad$ Uniform Computable Depth}

Bennett [4] defines an infinite sequence $A$ to be weakly deep if $A$ is not ttreducible to any algorithmically random sequence. The definition of algorithmic randomness, due to Martin-Löf [1], can be stated in terms of constructive null sets, which are sets with a uniformly computably enumerable sequence of open covers whose measures grow arbitrarily small. In this section we develop a similar notion of depth based on computable measure, a special case of the resource-bounded measure developed by Lutz [7]. This depth notion is used in the proof of our main result in Section 4. It is also of independent interest as it is closely related to Bennett's weak depth.

We first make our terminology precise. As in [12], we define a truth-table condition (briefly, a tt-condition) to be an ordered pair $\tau=\left(\left(n_{1}, \ldots, n_{k}\right), g\right)$, where $k, n_{1}, \ldots, n_{k} \in \mathbb{N}$ and $g:\{0,1\}^{k} \rightarrow\{0,1\}$. We write TTC for the class of all tt-conditions. The tt-value of a sequence $B \in \mathbf{C}$ under a tt-condition $\tau=\left(\left(n_{1}, \ldots, n_{k}\right), g\right)$ is the bit $\tau^{B}=g\left(B\left[n_{1}\right] B\left[n_{2}\right] \cdots B\left[n_{k}\right]\right)$. If $\tau$ is a ttcondition, then we say that $\tau$ queries the integer $m$ if $m \in\left\{n_{1}, \ldots, n_{k}\right\}$, and the query height of $\tau$ is defined as $\max \left(n_{1}, \ldots, n_{k}\right)+1$. 
A truth-table reduction (briefly, a tt-reduction) is a total computable function $F: \mathbb{N} \rightarrow$ TTC. A truth-table reduction $F$ naturally induces a function $\widehat{F}: \mathbf{C} \rightarrow$ C defined by

$$
\widehat{F}(B)=F(0)^{B} F(1)^{B} \cdots .
$$

In general, we identify a truth-table reduction $F$ with the induced function $\widehat{F}$, writing $F$ for either function and relying on context to avoid confusion.

The following terminology is convenient for our purposes.

Definition $4 A$ uniform reducibility is a total computable function $F: \mathbb{N} \times \mathbb{N} \rightarrow$ TTC.

If $F$ is a uniform reducibility, then we use the notation $F_{k}(n)=F(k, n)$ for all $k, n \in \mathbb{N}$. We thus regard a uniform reducibility as a computable sequence $F_{0}, F_{1}, F_{2}, \ldots$ of tt-reductions.

Definition 5 If $F$ and $G$ are uniform reducibilities, then we define the composition of $F$ with $G$ to be the uniform reducibility

$$
F \circ G: \mathbb{N} \times \mathbb{N} \rightarrow \mathrm{TTC}
$$

defined by

$$
(F \circ G)(\langle k, j\rangle, n)=\left(F_{k} \circ G_{j}\right)(n)
$$

for all $k, j, n \in \mathbb{N}$, where " $F_{k} \circ G_{j}$ " denotes the (easily defined) truth-table reduction satisfying $\left(F_{k} \circ G_{j}\right)(B)=F_{k}\left(G_{j}(B)\right)$ for all $B \in \mathbf{C}$.

Definition 6 Suppose $F$ is a uniform reducibility, and $A$ and $B$ are infinite binary sequences.

(1) $A$ is $F$-reducible to $B$, and we write $A \leq_{F} B$, if there is some $k$ such that $A=F_{k}(B)$.

(2) The upper $F$-span of $A$ is the set $F^{-1}(A)=\left\{X \in \mathbf{C}: A \leq_{F} X\right\}$.

(3) $A$ is computably $F$-deep if $\mu_{\text {comp }}\left(F^{-1}(A)\right)=0$.

(4) $A$ is computably weakly deep if, for every uniform reducibility $F, A$ is computably F-deep.

We pursue for a moment the analogy between Definition 6(3) and Bennett's weak depth. In [13], Terwijn and Torenvliet extended the resource-bounded measure of Lutz [7] using computably enumerable supermartingales, functions like martingales except the averaging condition they must satisfy is weaker than that required of ordinary martingales. Using this notion of measure, 
termed c.e. measure, Terwijn and Torenvliet proved that the class of nonalgorithmically random languages is the maximum c.e. measure 0 class. Bennett's notion of weak depth can thus be characterized in terms of c.e. measure in the sense that a language $A$ is weakly deep if and only if the c.e. measure of its upper tt-span is 0. Definition 6(3) reflects the spirit of this characterization, but replaces 'tt-reducible' with ' $F$-reducible' and replaces 'c.e. measure 0' with 'comp-measure 0'. Regarding Definition 6(4), observe that every computably weakly deep sequence is weakly deep. Lathrop and Lutz [14] have shown the converse is not true.

Although the definition of a weakly useful sequence was stated in terms of Turing reductions, we work almost exclusively in this section and the next with truth table reductions. The connection between these two notions is expressed by the following well-known fact.

Lemma 7 For every computable time bound $t(n)>0$, there is a uniform reducibility $F$ such that for all $x \in \mathbf{C}$, DTIME ${ }^{x}(t)=\left\{F_{0}(x), F_{1}(x), \ldots\right\}$.

We now prove the main result of this section.

Theorem 8 For every uniform reducibility $F$, almost every sequence in DEC is computably F-deep.

PROOF. Let $F$ denote a uniform reducibility, and write $F=F_{0}, F_{1}, \ldots$ For each $j \in \mathbb{N}$, define $D_{j}(w)=\left\{B \in \mathbf{C}: F_{j}(B)[0 \ldots|w|-1]=w\right\}$, i.e., those oracles which allow $F_{j}$ to correctly compute $w$. For every $j \in \mathbb{N}$ and $w \in\{0,1\}^{*}$, we may, using a program that computes $F$, calculate $L_{j}(w)=$ $\max \left(\left\{h \in \mathbb{N}: h\right.\right.$ is the query height of $F_{j}(i)$ for some $\left.\left.0 \leq i<|w|\right\}\right)$, and then poll $\{0,1\}^{L_{j}(w)}$ using the tt-conditions $F_{j}(i)$ for $0 \leq i<|w|$ to obtain the set

$$
E_{j}(w)=\left\{\alpha \in\{0,1\}^{L_{j}(w)}: F_{j}(B)[0 \ldots|w|-1]=w \text { for all } B \sqsupseteq \alpha\right\} .
$$

Let $\operatorname{Pr}\left(D_{j}(w)\right)$ denote the probability that an oracle chosen at random belongs to $D_{j}(w)$. Then the function $\widetilde{d}(w)=2^{|w|} \cdot \operatorname{Pr}\left(D_{j}(w)\right)$ is a martingale, and $\left\{\widetilde{d}_{j}\right\}_{j=0}^{\infty}$ is uniformly computable since $\operatorname{Pr}\left(D_{j}(w)\right)=\left|E_{j}(w)\right| \cdot 2^{-L_{j}(w)}$. Set

$$
\widetilde{d}(w)=\sum_{j=0}^{\infty} 2^{-j} \cdot \widetilde{d}_{j}(w) .
$$

It is routine to verify that $\tilde{d}$ is computable.

To show that $\mu_{\text {comp }}\left(F^{-1}(A)\right)=0$ for almost every $A \in$ DEC, we construct a computable martingale $d$ which succeeds on $F^{-1}(A)$ whenever $A \in$ DEC - 
$S^{\infty}[\widetilde{d}]$. Then for such an $A$ and for every $j \in \mathbb{N}$, we have

$$
\lim _{m \rightarrow \infty} \operatorname{Pr}\left(D_{j}(A[0 \ldots m])\right)=0
$$

Because of this we may, for each $j, n \in \mathbb{N}$, compute a number $m_{j, n}$ such that $\operatorname{Pr}\left(D_{j}\left(A\left[0 \ldots m_{j, n}\right]\right)\right) \leq 2^{-j-n-1}$. This can be accomplished by using programs that compute both $F$ and $A$ to calculate, for any $j, n \in \mathbb{N}, \operatorname{Pr}\left(D_{j}(A[0 \ldots m])\right)$ for increasing values of $m$. We then define $m_{j, n}$ to be the least $m$ such that $\operatorname{Pr}\left(D_{j}(A[0 \ldots m])\right) \leq 2^{-j-n-1}$. We remark that $\left\{m_{j, n}\right\}_{j, n=0}^{\infty}$ is uniformly computable, and define a uniformly computable sequence $\left\{d_{j, n}\right\}_{j, n=0}^{\infty}$ of martingales as follows. For all $j, n \in \mathbb{N}$, let $d_{j, n}$ be the unique martingale with initial value $d_{j, n}(\lambda)=\operatorname{Pr}\left(D_{j}\left(A\left[0 \ldots m_{j, n}\right]\right)\right)$, and satisfying $D_{j}\left(A\left[0 \ldots m_{j, n}\right]\right)=S S^{1}\left[d_{j, n}\right]$.

This implies that $d_{j, n}(\lambda) \leq 2^{-j-n-1}$ for all $j, n \in \mathbb{N}$, and we define a function $d:\{0,1\}^{*} \rightarrow[0, \infty)$ by

$$
d(w)=\sum_{n=0}^{\infty} \sum_{j=0}^{\infty} d_{j, n}(w) .
$$

Then

$$
d(\lambda)=\sum_{n=0}^{\infty} \sum_{j=0}^{\infty} d_{j, n}(\lambda) \leq \sum_{n=0}^{\infty} \sum_{j=0}^{\infty} 2^{-j-n-1}=2,
$$

and thus $d$ is a martingale. To see that it is computable, define $\hat{d}(r, w)=$ $\sum_{n=0}^{r+1+|w|} \sum_{j=0}^{r+1+|w|} d_{j, n}(w)$. The fact that $\left\{d_{j, n}\right\}_{j, n=0}^{\infty}$ is uniformly computable implies that $\hat{d}(r, w)$ is computable, and

$$
\begin{aligned}
|d(w)-\hat{d}(r, w)| & \leq \sum_{n=0}^{\infty} \sum_{j=r+2+|w|}^{\infty} 2^{|w|} \cdot 2^{-j-n-1}+\sum_{n=r+2+|w|}^{\infty} \sum_{j=0}^{\infty} 2^{|w|} \cdot 2^{-j-n-1} \\
& =\sum_{n=0}^{\infty} 2^{-r-2-n}+\sum_{n=r+2+|w|}^{\infty} 2^{|w|-n} \\
& =2^{-r-1}+2^{-r-1}=2^{-r} .
\end{aligned}
$$

For every $B \in F^{-1}(A)$, there exists $j \in \mathbb{N}$ such that for all $n \in \mathbb{N}, B \in$ $S S^{1}\left[d_{j, n}\right]$, whence $F^{-1}(A) \subseteq S^{\infty}[d]$. This shows that $\mu_{\text {comp }}\left(F^{-1}(A)\right)=0$. The sequence $A \in \mathrm{DEC}-S^{\infty}[\widetilde{d}]$ was arbitrary, so it follows that almost every decidable sequence is computably $F$-deep.

Theorem 9 Every weakly useful sequence is computably weakly deep. 
PROOF. Assume that $A$ is weakly useful and fix a uniform reducibility $F$. Fix a computable time bound $t: \mathbb{N} \rightarrow \mathbb{N}$ such that $\mu\left(\operatorname{DTIME}^{A}(t) \mid\right.$ DEC $) \neq 0$. Then by Lemma 7 there is a uniform reducibility $\widetilde{F}$ such that $\operatorname{DTIME}^{A}(t)=$ $\left\{\widetilde{F}_{0}(A), \widetilde{F}_{1}(A), \ldots\right\}$. Let $X$ denote the collection of computably $(\widetilde{F} \circ F)$-deep sequences. By Theorem $8, \mu(X \mid \mathrm{DEC})=1$, so there is a sequence $B \in X \cap$ $\operatorname{DTIME}^{A}(t) \cap$ DEC. Let $C \in F^{-1}(A)$ and choose $j, k \in \mathbb{N}$ such that $A=F_{j}(C)$ and $B=\widetilde{F}_{k}(A)$. Then $B=\widetilde{F}_{k}\left(F_{j}(C)\right)$, so $C \in(\widetilde{F} \circ F)^{-1}(B)$. This shows that $F^{-1}(A) \subseteq(\widetilde{F} \circ F)^{-1}(B)$. Since $B \in X$, it follows that $\mu_{\text {comp }}\left(F^{-1}(A)\right)=$ $\mu_{\text {comp }}\left((\widetilde{F} \circ F)^{-1}(B)\right)=0$, whence $A$ is computably weakly deep.

\section{Main Result}

In this section, we prove the existence of weakly useful sequences that are not strongly useful. Although we consider infinite, nonuniform collections of uniform reducibilities $F$, our construction uses computably $F$-deep sets that are constructed in a canonical way.

We will deal extensively with partial characteristic functions, i.e., functions with domain a subset of $\mathbb{N}$ and range $\{0,1\}$. If $\sigma$ and $\tau$ are partial characteristic functions, we let $\operatorname{dom}(\sigma)$ denote the domain of $\sigma$, and say that $\sigma$ and $\tau$ are compatible if they agree on all elements in $\operatorname{dom}(\sigma) \cap \operatorname{dom}(\tau)$. We say that $\sigma$ is extended by $\tau(\sigma \sqsubseteq \tau)$ if $\sigma$ and $\tau$ are compatible and $\operatorname{dom}(\sigma) \subseteq \operatorname{dom}(\tau)$. If $\mathcal{D} \subseteq \mathbb{N}$, then $\sigma$ restricted to $\mathcal{D}$ is the unique partial characteristic function

$$
\tau[x]= \begin{cases}\sigma[x] & \text { if } x \in \mathcal{D} \\ \text { undefined otherwise }\end{cases}
$$

We often identify $\mathbb{N}$ with $\mathbb{N}^{2}$ via the pairing function. The $i$ th section of natural numbers $\{\langle i, j\rangle: j \in \mathbb{N}\}$ is denoted by $\mathbb{N}_{i}$, the union of the first $i$ sections $\mathbb{N}_{0} \cup \ldots \cup \mathbb{N}_{i-1}$ by $\mathbb{N}_{<i}$, and the complement of $\mathbb{N}_{<i}$ by $\mathbb{N}_{\geq i}$. If $\sigma$ is a partial characteristic function and $n \in \mathbb{N}$, then

- $\sigma_{=i}$ denotes $\sigma$ restricted to the domain $\mathbb{N}_{i}$

- $\sigma_{=i}[<n]$ denotes $\sigma$ restricted to the domain $\{\langle i, y\rangle: 0 \leq y<n\}$,

- $\sigma_{<i}$ denotes $\sigma$ restricted to the domain $\mathbb{N}_{<i}$, and

- $\sigma_{<i}[<n]$ is $\sigma$ restricted to $\{\langle x, y\rangle: 0 \leq x<i$ and $0 \leq y<n\}$.

Definition 10 For each uniform reducibility $F$, the decidable sequence A produced by the construction below is called the canonical computably F-deep sequence.

Construction. Suppose $F$ is a uniform reducibility. Fix $j, n \in \mathbb{N}$, and let $L$ 
be the maximum of the query heights of $F_{j}\left(\left\langle j, n^{\prime}\right\rangle\right)$ for all $n^{\prime} \leq n$. Partition $\{0,1\}^{L}$ into two sets $R_{0}$ and $R_{1}$ so that

$$
\alpha \in R_{b} \Longleftrightarrow\left(F_{j}(\langle j, n\rangle)^{B}=b \text { for every oracle } B \sqsupseteq \alpha\right) \text {. }
$$

Informally, we identify $R_{0}$ with the set of oracles which answer "No" when queried by $F_{j}(\langle j, n\rangle)$, and $R_{1}$ with the set of oracles which answer "Yes." Our strategy will be to diagonalize against the majority in the construction of $A$, ensuring that only the minority answer among those consistent with previous answers can correctly compute any given bit of $A$. We thus define $A[\langle j, n\rangle]$ by induction as follows. Assume that $A\left[\left\langle j, n^{\prime}\right\rangle\right]$ has already been defined for all $n^{\prime}<n$, and let

$$
R=\left\{\alpha \in\{0,1\}^{L}:(\forall B \sqsupseteq \alpha)\left(A_{=j}[<n] \sqsubseteq F_{j}(B)\right)\right\}
$$

consist of the strings from which $F_{j}$ computes the previous values of $A$ on the $j$ th section correctly. Then we define

$$
A[\langle j, n\rangle]=\left\{\begin{array}{l}
1 \text { if }\left|R_{1} \cap R\right| \leq\left|R_{0} \cap R\right| \\
0 \text { if }\left|R_{0} \cap R\right|<\left|R_{1} \cap R\right|
\end{array}\right.
$$

Clearly $A$ is decidable. Furthermore, our definition ensures that the partial functions $A_{=j}[<n]$ of $A$ have the following property.

Fact 11 The probability that an oracle chosen at random allows $F_{j}$ to correctly determine $A_{=j}[<n+1]$ is at most half the probability that it allows $F_{j}$ to correctly determine $A_{=j}[<n]$.

It only remains to show that $A$ is computably $F$-deep. To this end, define

$$
D_{j, n}=\left\{B \in \mathbf{C}: A_{=j}[<n] \sqsubseteq F_{j}(B)\right\} .
$$

The computation of $A_{=j}[<n]$ is accomplished without making queries to any $k \geq L$, hence there is some $S \subseteq\{0,1\}^{L}$ such that

$$
D_{j, n}=\bigcup_{w \in S} \mathbf{C}_{w}
$$

As in the proof of Theorem 8, we define $d_{j, n}$ to be the unique martingale with initial value $d_{j, n}(\lambda)=\operatorname{Pr}\left(D_{j, n}\right)$ and such that $D_{j, n}=S S^{1}\left[d_{j, n}\right]$. By Fact 11 we have $d_{j, n+1}(\lambda) \leq \frac{1}{2} d_{j, n}(\lambda)$, and thus $d_{j, n}(\lambda) \leq 2^{-n}$ for any $n \in \mathbb{N}$. 
Define $d:\{0,1\}^{*} \rightarrow[0, \infty)$ by

$$
d(w)=\sum_{j=0}^{\infty} \sum_{n=0}^{\infty} 2^{-j} d_{j, n}(w) .
$$

Then

$$
d(\lambda) \leq \sum_{j=0}^{\infty} \sum_{n=0}^{\infty} 2^{-j-n}=4
$$

so $d$ is a martingale. If we define $\hat{d}: \mathbb{N} \times\{0,1\}^{*} \rightarrow[0, \infty)$ by

$$
\hat{d}(r, w)=\sum_{j=0}^{r+|w|+2} \sum_{k=0}^{r+|w|+2} 2^{-j} d_{j, n}(w),
$$

then $\hat{d}$ is computable. Also, each $d_{j, n}(w) \leq 2^{|w|} d_{j, n}(\lambda) \leq 2^{|w|-n}$, so for all $r \in \mathbb{N}$ and $w \in\{0,1\}^{*}$,

$$
\begin{aligned}
|\hat{d}(r, w)-d(w)| & \leq \sum_{j=r+|w|+3}^{\infty} \sum_{n=0}^{\infty} 2^{|w|-j-n}+\sum_{j=0}^{\infty} \sum_{n=r+|w|+3}^{\infty} 2^{|w|-j-n} \\
& =2 \cdot \sum_{j=r+|w|+3}^{\infty} \sum_{n=0}^{\infty} 2^{|w|-j-n} \\
& =4 \cdot \sum_{j=r+|w|+3}^{\infty} 2^{|w|-j} \\
& =2^{-r}
\end{aligned}
$$

i.e., $\hat{d}$ testifies that $d$ is computable.

We finish the proof by showing that $F^{-1}(A) \subseteq S^{\infty}[d]$. To see this, let $B \in$ $F^{-1}(A)$, and fix $j \in \mathbb{N}$ such that $A=F_{j}(B)$. Then $B \in \bigcap_{n=0}^{\infty} D_{j, n}=$ $\cap_{n=0}^{\infty} S S^{1}\left[d_{j, n}\right]$, so for every $m \in \mathbb{N}$ there is a $w \sqsubseteq B$ such that

$$
\begin{aligned}
d(w) & \geq 2^{-j} \sum_{n=0}^{m 2^{j}-1} d_{j, n}(w) \\
& \geq 2^{-j} \sum_{n=0}^{m 2^{j}-1} 1 \\
& =m .
\end{aligned}
$$

This shows that $B \in S^{\infty}[d]$. We now have a computable martingale $d$ with $F^{-1}(A) \subseteq S^{\infty}[d]$. 
Thus we have proved the following.

Proposition 12 If $F$ is a uniform reducibility and $A$ is the canonical computably $F$-deep sequence, then $\mu_{\text {comp }}\left(F^{-1}(A)\right)=0$.

We are now ready to prove the main theorem. Our proof is an adaptation of the martingale diagonalization method introduced by Lutz in [11]. We define a sequence $H$ one section at a time to satisfy the following conditions, where $H_{0}, H_{1}, H_{2}, \ldots$ are the sections of $H$, i.e., $H_{k}[n]=H[\langle k, n\rangle]$.

(1) Each section $H_{k}$ is decidable (although $H$ itself cannot be decidable).

(2) If $d$ is any computable martingale, then there is some $k$ such that $d$ fails on $H_{k}$.

(3) For every computable time bound $t$, there is a decidable set which is not in $\operatorname{DTIME}^{H}(t)$.

These three conditions suffice for our purposes. By Condition 1, the set $J=$ $\left\{H_{0}, H_{1}, H_{2}, \ldots\right\} \subseteq$ DEC, and by Condition 2, no computable martingale can succeed on all its elements. Thus $\mu_{\text {comp }}(J) \neq 0$, which makes $H$ weakly useful, since $J \subseteq$ DTIME $^{H}$ (linear). Condition 3 ensures that $H$ is not strongly useful.

Theorem 13 There exists a sequence $H$ that is weakly useful but not strongly useful.

PROOF. We divide the proof into two sections: (i) the construction of $H$, and (ii) the proof that $H$ satisfies the three conditions above, and is thus weakly useful but not strongly useful.

\section{Construction of $H$}

Fix an arbitrary enumeration $t_{0}, t_{1}, \ldots$ of all computable time bounds and an enumeration $\tilde{d}_{0}, \tilde{d}_{1}, \ldots$ of all computable martingales. These enumerations need not be uniform in any sense, since we are not trying to control the complexity of $H$. We will define (in order) a number of different objects for each $k$ :

- a uniform reducibility $F^{k}$ corresponding to $t_{k}$,

- a decidable $A^{k}$ such that $A^{k} \notin \operatorname{DTIME}^{H}\left(t_{k}\right)$,

- a partial characteristic function $\alpha_{k}$ of finite domain, compatible with all the previous sections of $H$,

- martingales $d_{k, q}^{i, j}$ which are uniformly computable over $i, j, q \in \mathbb{N}$, which, taken together for all $k \geq i$, witness that each $A^{i}$ is computably $F^{i}$-deep, and

- the section $H_{k}$ itself, which is designed to make the martingale 


$$
d_{k}=\tilde{d}_{k}+\sum_{i=0}^{k} \sum_{j=0}^{\infty} \sum_{q=0}^{\infty} d_{k, q}^{i, j} \cdot 2^{-q-j}
$$

fail on $H_{k}$, thus satisfying Condition 2 above. The section $H_{k}$ will also participate in a fixed finite number of diagonalizations against tt-reductions from $A^{i}$ to $H$ for all $i \leq k$.

Fix $k \in \mathbb{N}$ and assume the above objects have been defined for all $k^{\prime}<k$ (define $\alpha_{-1}=\lambda$ ). Also assume that for each $k^{\prime}<k$ we have at our disposal programs to compute the objects $A^{k^{\prime}}$ and $F_{j}^{k^{\prime}}$ uniformly over $j$. Let

$$
M_{0}^{k}, M_{1}^{k}, \ldots
$$

be a computable enumeration of all oracle Turing machines running in time $t_{k}$, and let

$$
\widehat{M}_{0}^{k}, \widehat{M}_{1}^{k}, \ldots
$$

be a computable enumeration of oracle Turing machines which behave exactly like the corresponding $M_{j}^{k}$ 's, except that when $M_{j}^{k}$ makes a query of the form $\langle x, y\rangle$ for $x<k, \widehat{M}_{j}^{k}$ instead simulates the answer by computing $H_{x}[y]$ directly. Let

$$
F_{0}^{k}, F_{1}^{k}, \ldots
$$

be an enumeration of tt-reductions, each of which simulates the corresponding $\widehat{M}_{m}^{k}$. This enumeration is computable and thus is a uniform reducibility. Note that on any input, $F_{j}^{k}$ only makes queries to $\mathbb{N}_{\geq k}$.

We define $A^{k}$ to be the canonical computably $F^{k}$-deep set. Let $r, s \in \mathbb{N}$ be the unique pair such that $k=\langle r, s\rangle$. If there is a total characteristic function $B$ extending both $H_{<k}$ and $\alpha_{k-1}$ for which $A^{r} \neq F_{s}^{r}(B)$, then let $\alpha_{k} \sqsupseteq \alpha_{k-1}$ be a finite sequence (the lexicographic minimum, say) that is compatible with $H_{<k} \cup \alpha_{k-1}$ and whose domain is big enough to preserve this fact, i.e., $A^{r} \neq$ $F_{s}^{r}\left(B^{\prime}\right)$ for any $B^{\prime} \in \mathbf{C}$ such that $B^{\prime} \sqsupseteq \alpha_{k}$. In this case we say $\alpha_{k}$ diagonalizes against $F_{s}^{r}$. Otherwise, we let $\alpha_{k}=\alpha_{k-1}$. This finite sequence will eventually be included as a subseqence of $H$, but is added just one section at a time.

Now fix any $i, j \in \mathbb{N}$ with $i \leq k$. For each $n \in \mathbb{N}$ and $w \in\{0,1\}^{*}$, we define

(1) $D_{j, n}^{i}=\left\{B \in \mathbf{C}: A_{=j}^{i}[<n] \sqsubseteq F_{j}^{i}(B)\right\}$,

(2) $R_{j, n}=\left\{B \in \mathbf{C}: H_{<j}[<n] \sqsubseteq B\right\}$, and 


$$
S_{j, w}=\{B \in \mathbf{C}:(\forall 0 \leq m<|w|)(B[\langle j, m\rangle]=w[m])\} .
$$

Fact 11 implies that $\operatorname{Pr}\left(D_{j, n}^{i}\right) \leq 2^{-n}$, and it is easy to see that $\operatorname{Pr}\left(R_{j, n}\right)=2^{-j n}$. Put

$$
y_{i, j}(n)=\min \left\{y \in \mathbb{N}: \begin{array}{l}
\text { for all } 0 \leq m<n, \text { all queries of } F_{j}^{i}(\langle j, m\rangle) \\
\text { are of the form }\left\langle x, y^{\prime}\right\rangle \text { with } y^{\prime}<y
\end{array}\right\} .
$$

For all $q, \ell \in \mathbb{N}$, define (letting $Y=R_{k, y_{i, j}(q \ell)} \cap D_{j, q \ell}^{i}$ )

$$
d_{k, q, \ell}^{i, j}(w)= \begin{cases}2^{|w|-\ell} \cdot \operatorname{Pr}\left(S_{k, w} \mid Y\right) & \text { if } \operatorname{Pr}(Y)>0 \\ 2^{-\ell} & \text { otherwise }\end{cases}
$$

where the probabilities refer to the uniform probability measure on $\mathbf{C}$, and, for measurable sets $X, Y \subseteq \mathbf{C}$ with $\operatorname{Pr}(Y)>0$, we define $\operatorname{Pr}(X \mid Y)$ to be $\operatorname{Pr}(X \cap Y) / \operatorname{Pr}(Y)$ as usual. Note that the definition of $d_{k, q, \ell}^{i, j}$ above remains unchanged if we replace $y_{i, j}(q \ell)$ with any $y \geq y_{i, j}(q \ell)$, because $D_{j, q \ell}^{i}$ depends on $B \in \mathbf{C}$ only for those queries made by $F_{j}^{i}$ on inputs $\langle j, 0\rangle, \ldots,\langle j, q \ell-1\rangle$, and none of these queries is of the form $\langle x, y\rangle$ for $y \geq y_{i, j}(q \ell)$.

Summing over $\ell \geq 1$, define

$$
d_{k, q}^{i, j}(w)=\sum_{\ell=1}^{\infty} d_{k, q, \ell}^{i, j}(w) .
$$

Finally, set

$$
d_{k}(w)=\tilde{d}_{k}(w)+\sum_{i=0}^{k} \sum_{j=0}^{\infty} \sum_{q=0}^{\infty} d_{k, q}^{i, j}(w) \cdot 2^{-q-j} .
$$

We define $H_{=k}: \mathbb{N}_{k} \rightarrow\{0,1\}$ so that it is compatible with $\alpha_{k}$ on their common domain but diagonalizes out of the success set of $d_{k}$ otherwise. Specifically,

$$
H_{k}[n]=H_{=k}[\langle k, n\rangle]= \begin{cases}\alpha_{k}[\langle k, n\rangle] & \text { if it is defined } \\ \llbracket d_{k}\left(H_{k}[<n] 1\right) \leq d_{k}\left(H_{k}[<n] 0\right) \rrbracket \text { otherwise. }\end{cases}
$$

As stated, it may not be the case that the comparison in the definition can actually be accomplished since a computable martingale such as $d_{k}$ cannot in general be computed exactly, but is only approximated. What we are really comparing then are not $d_{k}\left(H_{k}[<n] 1\right)$ and $d_{k}\left(H_{k}[<n] 0\right)$, but rather their $n$th approximations, which are computable. Since these approximations are 
guaranteed to be within $2^{-n}$ of the actual values, and our sole aim is to make $d_{k}$ fail on $H_{k}$, it suffices for our purposes to consider only the approximations when doing the comparisons above. The same trick is used in [11].

$H_{k}$ is decidable, and for cofinitely many $n, H_{k}[n]$ is chosen so that $d_{k}\left(H_{k}[<\right.$ $(n+1)]) \leq d_{k}\left(H_{k}[<n]\right)+2^{-n}$, the $2^{-n}$ owing to the error in the approximation of $d_{k}$. Thus $d_{k}$ fails on $H_{k}$, from which we obtain

Fact 14 The martingales $\tilde{d}_{k}$ and $d_{k, q}^{i, j}$, where $j, q$ are arbitrary and $i \leq k$, all fail on $H_{k}$.

Thus Conditions 1 and 2 above are satisfied. Define $H$ to be the function whose value at $\langle u, v\rangle$ is $H_{u}[v]$. Each $H_{k}$ preserves the diagonalization commitments made by the $\alpha_{k^{\prime}}$ for $k^{\prime} \leq k$, and one can easily see that $\alpha_{0} \sqsubseteq \alpha_{1} \sqsubseteq \cdots H$. This completes the construction of $H$.

\section{$H$ is weakly useful but not strongly useful}

Notice that $J=\left\{H_{0}, H_{1}, \ldots\right\} \subseteq \operatorname{DTIME}^{H}$ (linear), but $\mu_{\text {comp }}(J) \neq 0$. Therefore, $H$ is weakly useful. It only remains to show that $H$ is not strongly useful. For every $i, \operatorname{DTIME}^{H}\left(t_{i}\right)=\left\{B \in \mathbf{C}: B \leq_{F^{i}} H\right\}$. Hence it suffices to show that for all $i$ and $j$, the canonical computably $F^{i}$-deep set $A^{i} \neq F_{j}^{i}(H)$.

We assume there are natural numbers $r$ and $s$ such that $A^{r}=F_{s}^{r}(H)$ and work towards a contradiction. Define $k_{0}=\langle r, s\rangle$ and let $\sigma=H_{<k_{0}} \cup \alpha_{k_{0}-1}$. By the definition of $\alpha_{k_{0}}$, it must be the case that $A^{r}=F_{s}^{r}(B)$ for every $B \sqsupseteq \sigma$, otherwise $F_{s}^{r}$ would have been diagonalized against by $\alpha_{k_{0}}$ and would thus fail to reduce $A^{r}$ to $H$. Choose $q_{0}>r$ such that $\mathbb{N}_{\geq q_{0}} \cap \operatorname{dom}(\sigma)=\emptyset$. We will show that $d_{n, q_{0}}^{r, s}$ succeeds on $H_{n}$ for some $n<q_{0}$, contradicting Fact 14 .

For every $y \in \mathbb{N}$, let $\left\{\delta_{i}: 0 \leq i<2^{r y}\right\}$ be an enumeration of all partial characteristic functions with domain $\mathcal{T}=\{\langle u, v\rangle: 0 \leq u<r$ and $0 \leq v<y\}$, and such that $\delta_{0}=H_{<r}[<y]$. Partition $\mathbf{C}$ into cells $E_{i}=\left\{B \in \mathbf{C}: \delta_{i} \sqsubseteq B\right\}$. Fix any integer $\ell \geq 1$. Since $F_{s}^{r}$ makes no queries in $\mathcal{T}$, the probabilities $\operatorname{Pr}\left(D_{s, q_{0} \ell}^{r} \mid E_{i}\right)$ are all equal for $i<2^{r y}$. Therefore

$$
\operatorname{Pr}\left(D_{s, q_{0} \ell}^{r}\right)=\sum_{n=0}^{2^{r y}-1} \operatorname{Pr}\left(E_{n}\right) \cdot \operatorname{Pr}\left(D_{s, q_{0} \ell}^{r} \mid E_{n}\right)=2^{-r y} \sum_{n=0}^{2^{r y}-1} \operatorname{Pr}\left(D_{s, q_{0} \ell}^{r} \mid E_{0}\right)=\operatorname{Pr}\left(D_{s, q_{0} \ell}^{r} \mid R_{r, y}\right),
$$

and it follows that $R_{r, y}$ and $D_{s, q_{0} \ell}^{r}$ are independent for all $y$ and $\ell$. If $r \leq n<q_{0}$, then for $y \geq y_{r, s}\left(q_{0} \ell\right)$,

$$
\operatorname{Pr}\left(R_{n, y} \cap D_{s, q_{0} \ell}^{r}\right) \geq \operatorname{Pr}\left(R_{q_{0}, y} \cap D_{s, q_{0} \ell}^{r}\right)=\operatorname{Pr}\left(R_{q_{0}, y}\right)=2^{-q_{0} y}>0,
$$

since $R_{q_{0}, y} \subseteq D_{s, q_{0} \ell}^{r}$ by our assumption. For these $n$, only the top equation in 
(1) is relevant. Thus for sufficiently large $y$,

$$
\begin{aligned}
\prod_{n=r}^{q_{0}-1} d_{n, q_{0}, \ell}^{r, s}\left(H_{n}[<y]\right) & =\prod_{n=r}^{q_{0}-1} 2^{y-\ell} \cdot \operatorname{Pr}\left(S_{n, H_{n}[<y]} \mid R_{n, y} \cap D_{s, q_{0} \ell}^{r}\right) \\
& =2^{(y-\ell)\left(q_{0}-r\right)} \cdot \prod_{n=r}^{q_{0}-1} \operatorname{Pr}\left(R_{n, y} \cap S_{n, H_{n}[<y]} \mid R_{n, y} \cap D_{s, q_{0} \ell}^{r}\right) \\
& =2^{(y-\ell)\left(q_{0}-r\right)} \cdot \prod_{n=r}^{q_{0}-1} \operatorname{Pr}\left(R_{n+1, y} \mid R_{n, y} \cap D_{s, q_{0} \ell}^{r}\right) \\
& =2^{(y-\ell)\left(q_{0}-r\right)} \cdot \operatorname{Pr}\left(R_{q_{0}, y} \mid R_{r, y} \cap D_{s, q_{0} \ell}^{r}\right) \\
& =2^{(y-\ell)\left(q_{0}-r\right)} \cdot \frac{\operatorname{Pr}\left(R_{q_{0}, y} \cap D_{s, q_{0} \ell}^{r}\right)}{\operatorname{Pr}\left(R_{r, y}\right) \operatorname{Pr}\left(D_{s, q_{0} \ell}^{r}\right)} .
\end{aligned}
$$

By Fact 11, $\operatorname{Pr}\left(D_{s, q_{0} \ell}^{r}\right) \leq 2^{-q_{0} \ell}$, and this together with (2) implies that the last expression above is bounded from below by

$$
\frac{2^{q_{0} y-q_{0} \ell-r y+r \ell} \cdot 2^{-q_{0} y}}{2^{-r y} \cdot 2^{-q_{0} \ell}}=2^{r \ell} \geq 1
$$

Hence for any $\ell$ and all $y \geq y_{r, s}\left(q_{0} \ell\right)$, there must exist some $n$ satisfying $r \leq n<q_{0}$ and $d_{n, q_{0}, \ell}^{r, s}\left(H_{n}[<y]\right) \geq 1$. Then by the Pigeon Hole Principle there is some $r \leq n_{0}<q_{0}$ with the property that, for infinitely many $\ell$, $d_{n_{0}, q_{0}, \ell}^{r, s}\left(H_{n_{0}}[<y]\right) \geq 1$ for all $y \geq y_{r, s}\left(q_{0} \ell\right)$. Hence $d_{n_{0}, q_{0}}^{r, s}=\sum_{\ell=1}^{\infty} d_{n_{0}, q_{0}, \ell}^{r, s}$ succeeds on $H_{n_{0}}$, but then so does $d_{n_{0}}$-a contradiction. Therefore, $H$ is not strongly useful.

Corollary 15 There is a sequence that is strongly deep but not strongly useful.

PROOF. This follows immediately from Theorem 13 and the fact [6] that every weakly useful sequence is strongly deep.

It is easy to verify that weak and strong usefulness are both invariant under ttequivalence. Thus, Theorem 13 shows that there are weakly useful tt-degrees that are not strongly useful. Our results do not say anything regarding the Turing degrees of weakly useful sets, however. In particular, we leave open the question of whether there is a weakly useful Turing degree that is not strongly useful (i.e., whether there is a weakly useful set not Turing equivalent to any strongly useful set). Some facts are known about these degrees. Jockusch [9] neatly characterized the strongly useful Turing degrees (under a different name), for example, as being either high or containing complete extensions of first-order Peano arithmetic. This includes some low degrees, but no non-high 
c.e. degrees. Stephan [15] has partially strengthened these results, showing that no non-high c.e. Turing degree can be weakly useful, either. Therefore, among the c.e. degrees, the strongly useful, weakly useful, and high degrees all coincide.

\section{Acknowledgements}

The authors thank Martin Kummer and Frank Stephan for helpful discussions.

\section{References}

[1] P. Martin-Löf, On the definition of random sequences, Information and Control 9 (1966) 602-619.

[2] Y. Barzdin', Complexity of programs to determine whether natural numbers not greater than $n$ belong to a recursively enumerable set, Soviet Mathematics Doklady 9 (1968) 1251-1254.

[3] E. L. Post, Recursively enumerable sets of positive integers and their decision problems, Bulletin of the American Mathematical Society 50 (1944) 284-316.

[4] C. H. Bennett, Logical depth and physical complexity, in: R. Herken (Ed.), The Universal Turing Machine: A Half-Century Survey, Oxford University Press, 1988, pp. 227-257.

[5] M. Li, P. M. B. Vitányi, An Introduction to Kolmogorov Complexity and its Applications, 2nd Edition, Springer, 1997.

[6] D. W. Juedes, J. I. Lathrop, J. H. Lutz, Computational depth and reducibility, Theoretical Computer Science 132 (1994) 37-70.

[7] J. H. Lutz, Almost everywhere high nonuniform complexity, Journal of Computer and System Sciences 44 (1992) 220-258.

[8] G. E. Sacks, Degrees of Unsolvability, Princeton University Press, 1966.

[9] C. G. Jockusch, Jr, Degrees in which the recursive sets are uniformly recursive, Canadian Journal of Mathematics 24 (1972) 1092-1099.

[10] C. G. Jockusch, Jr, R. I. Soare, Degrees of members of $\Pi_{1}^{0}$ classes, Pacific Journal of Mathematics 40 (1972) 605-616.

[11] J. H. Lutz, Weakly hard problems, SIAM Journal on Computing 24 (1995) 1170-1189.

[12] H. Rogers, Jr, Theory of Recursive Functions and Effective Computability, McGraw-Hill, 1967. 
[13] S. A. Terwijn, L. Torenvliet, Arithmetical measure, Mathematical Logic Quarterly 44 (4) (1998) 277-286.

[14] J. I. Lathrop, J. H. Lutz, Recursive computational depth, Information and Computation 153 (1999) 139-172.

[15] F. Stephan, personal communication (1994). 